Cahiers
de la Recherche
surles
$D$ roits Fondamentaux

\section{Cahiers de la recherche sur les droits} fondamentaux

15 | 2017

Le corps humain saisi par le droit : entre liberté et propriété

\title{
Le corps mis à disposition : la gestation pour autrui
}

The Body Made Available: Gestational Surrogacy

Armelle Gosselin-Gorand et Laurence Mauger-Vielpeau

\section{(2) OpenEdition}

Journals

Édition électronique

URL : https://journals.openedition.org/crdf/552

DOI : $10.4000 /$ crdf.552

ISSN : 2264-1246

Éditeur

Presses universitaires de Caen

Édition imprimée

Date de publication : 1 novembre 2017

Pagination : 77-85

ISBN : 978-2-84133-858-0

ISSN : $1634-8842$

Référence électronique

Armelle Gosselin-Gorand et Laurence Mauger-Vielpeau, «Le corps mis à disposition : la gestation pour autrui », Cahiers de la recherche sur les droits fondamentaux [En ligne], 15 | 2017, mis en ligne le 01 octobre 2019, consulté le 16 novembre 2022. URL : http://journals.openedition.org/crdf/552 ; DOI : https://doi.org/10.4000/crdf.552 


\title{
Le corps mis à disposition : la gestation pour autrui
}

\author{
Armelle GOSSELIN-GORAND \\ Maître de conférences (HDR) en droit privé à l'université de Caen Normandie \\ Institut Demolombe (EA 967) \\ Laurence MAUGER-VIELPEAU \\ Professeure de droit privé à l'université de Caen Normandie \\ Institut Demolombe (EA 967)
}

I. La liberté
II. La propriété

Ce phénomène, quel que soit le nom qu'on lui donne, a d'abord été porté par la procréation médicalement assistée, laquelle reste tributaire de la régulation par les pouvoirs publics de l'exercice de la médecine et on est dans ce domaine confronté à un monopole de la loi territoriale qui fixe les conditions de réalisation de la procréation par les personnels médicaux compétents. Puis, il a pris une autre dimension avec l'admission dans certains pays d'une privatisation du mécanisme de procréation grâce à la technique du contrat. C'est ce que l'on a appelé le contrat de gestation pour autrui, le contrat de mère porteuse ou encore le contrat de maternité de substitution.

À ce sujet, deux remarques doivent être faites. D'une part, ces contrats illustrent le fait que la gestation pour autrui est en réalité plurielle et que ce sont des gestations pour autrui dont il faut parler. Le corps entre propriété et liberté, le corps n'est pas traité de la même manière selon les cas. Les techniques dites de "mères porteuses», ou de «location d'utérus», appelées aujourd'hui plus volontiers gestation pour autrui ou maternité de substitution, recouvrent des pratiques diversifiées. Ainsi, l'enfant peut ne pas être génétiquement du couple d'accueil: la mère biologique donne ou vend l'enfant qu'elle a conçu avec son propre compagnon ou avec le sperme d'un tiers et qu'elle a porté (hypothèse de la maternité de substitution). Ensuite, l'enfant peut aussi être conçu par insémination de la mère porteuse avec le sperme du père biologique (marié ou pas): l'enfant est alors l'enfant biologique de cet homme, mais pas de son épouse ou de la femme avec qui il vit et la mère porteuse en est la mère biologique. Enfin, l'enfant peut même être génétiquement du couple d'accueil, la femme qui accouche ayant accepté de porter l'embryon issu des gamètes du couple d'accueil (hypothèse de la gestation pour autrui). Ces différences sont à souligner dès lors qu'est envisagée cette thématique, le corps n'étant pas concerné de la même manière dans chaque hypothèse, car on peut se demander s'il est raisonnable de traiter le sujet de manière globale. Nous en doutons fortement. D'autre part, ces contrats illustrent l'existence d'arrangements privés, qui supposent un positionnement des États identifiés comme prohibitifs ou permissifs, mais ils peuvent aussi inévitablement réintroduire des questions de droit international privé dès lors que tous les ordres juridiques ne se positionnent pas de la même manière et que les sujets de droit utilisent ces différences dans un contexte de développement des situations transfrontières. À partir du moment où des pays admettent le contrat de gestation pour autrui, le juriste, même lorsqu'il est issu d'un pays prohibitif, se trouve nécessairement confronté à des opérations triangulaires entre des couples issus des États non permissifs et une mère porteuse issue d'un pays permissif. 
Ces difficultés se sont accentuées puisque les positionnements sont variés en droit comparé et les moyens de déplacement suffisamment développés et aisés aujourd'hui pour que des couples aillent chercher ailleurs ce qui leur est interdit dans le for. On constate l'existence de mouvements transfrontières dans le domaine de la procréation, certains auteurs s'appuyant sur ce que l'on a appelé «les bébés Thalys » pour envisager dans le domaine du droit le phénomène d'un «tourisme procréatif » ou de migrations procréatives.

Pourtant, la France s'est positionnée sur ce sujet et constitue un État prohibitif. En droit interne, il n'y a pas de liberté, ni de propriété: il y a un interdit de la gestation pour autrui et de la maternité de substitution réalisées sur le territoire français.

Ce fut d'abord l'œuvre de la jurisprudence. Confrontée au développement sans précédent des techniques d'aide à la procréation, celle-ci a décidé très vite de poser des limites, suivant en cela une doctrine qui leur était déjà très hostile ${ }^{1}$. Pour ce faire, la Cour de cassation a d'abord estimé en 1989, dans l'arrêt Alma mater, que l'association qui a pour objet de faciliter la résolution des problèmes qui se posent aux couples dont la femme est stérile, et qui favorise la conclusion et l'exécution d'une convention entre ces couples et les mères porteuses volontaires, était illicite. Dans l'arrêt de l'assemblée plénière du 31 mai 1991, arrêt fortement attendu à l'époque d'autant que la solution affirmée en $1989^{2}$ se heurtait à une forte résistance des juges du fond, la Cour de cassation a ensuite déclaré illicite «la convention par laquelle une femme s'engage, fût-ce à titre gratuit, à concevoir et à porter un enfant pour l'abandonner à sa naissance ${ }^{3}$. L'arrêt est d'autant plus remarquable que le pourvoi était formé dans l'intérêt de la loi (situation tout à fait exceptionnelle), et après audition du président du Conseil consultatif national d'éthique, entendu comme amicus curiae. Les motifs de la nullité sont précisés: la convention «contrevient tant au principe d'ordre public de l'indisponibilité du corps humain qu'à celui de l'indisponibilité de l'état des personnes». Puis le législateur, dans le cadre des lois de bioéthique du 29 juillet 1994, a consacré partiellement cette jurisprudence : il est désormais affirmé solennellement à l'article 16-7 du Code civil que « [t] oute convention portant sur la procréation ou la gestation pour le compte d'autrui est nulle». Cette interdiction concerne toutes les conventions, que la mère porteuse soit mère génétique ou seulement gestatrice, qu'elle soit conclue à titre onéreux ou à titre gratuit. La prohibition a, plus tard ${ }^{4}$, été renforcée par la règle autorisant le ministère public à agir en nullité des reconnaissances en cas de fraude à la loi ${ }^{5}$. Cette interdiction est prolongée en droit pénal ${ }^{6}$. Depuis le Code pénal de $1992^{7}$, l'interdit de la maternité de substitution et de la gestation pour le compte d'autrui fait l'objet d'une section spécifique intitulée «Des atteintes à la filiation», sanctionnant les atteintes à l'indisponibilité de l'état d'un enfant, qui sont aussi à inscrire dans le contexte du respect des droits de l'enfant. Ces atteintes sont clairement dissociées de celles affectant la personne même de l'enfant, et de nature à compromettre sa santé ou sa sécurité ${ }^{8}$. En amont, est incriminée la provocation, adressée à des parents, ou l'un d'eux, à abandonner leur enfant né ou à naître, par don, promesse, menace ou abus d'autorité (art. 22712, al. $1^{\text {er }}$ du Code pénal: 6 mois d'emprisonnement et 7500 euros d'amende) ainsi que le fait de s'entremettre, dans un but lucratif, entre une personne ou un couple désireux d'accueillir un enfant et un parent souhaitant abandonner son enfant, ou une femme acceptant de porter pour eux un enfant (art. 227-12, al. 2 et 3 du Code pénal: 1 an d'emprisonnement et 15000 euros d'amende; les peines sont portées au double si les faits sont commis de façon habituelle et la tentative est punie des mêmes peines). L'article 227-13 du Code pénal incrimine les actes tendant à rattacher l'enfant officiellement aux personnes qui souhaitent se faire passer pour ses parents biologiques. Ces actes caractérisent les délits de «simulation ou dissimulation ayant entraîné une atteinte à l'état civil

1. Voir notamment P. Raynaud, «L'enfant peut-il être objet de droit?», Recueil Dalloz, 1988, chron., p. 109 sq.; P. Kayser, «Les limites morales et juridiques de la procréation artificielle», Recueil Dalloz, 1987, chron., p. 190 sq.

2. Sur laquelle, voir Les grands arrêts de la jurisprudence civile, H. Capitant, F. Terré, Y. Lequette (dir.), 13 éd., Paris, Dalloz, 2015, t. I, nº 51. Voir $^{\mathrm{e}}$ aussi A. Batteur, A. Cerf-Hollender, A. Gosselin-Gorand, J.-M. Larralde, «Les interdits de la maternité de substitution et de gestation pour le compte d'autrui ", in Les grandes décisions du droit des personnes et de la famille, A. Batteur (dir.), 2 éd., Issy-les-Moulineaux, LGDJ, 2016, p. 212 sq.

3. Cass., Ass. plén., 31 mai 1991, Alma mater, nº 90-20.105, publié au Recueil Lebon. Le caractère illicite de la convention avait déjà été affirmé en des termes approchants dans l'arrêt du 13 décembre 1989.

4. Ordonnance $\mathrm{n}^{\circ} 2005-759$ du 4 juillet 2005 portant réforme de la filiation, Journal officiel de la République française, $\mathrm{n}^{\circ} 156,6$ juillet 2005 , p. 11159

5. Art. $336 \mathrm{du}$ Code civil: «La filiation légalement établie peut être contestée par le ministère public si des indices tirés des actes eux-mêmes la rendent invraisemblable ou en cas de fraude à la loi».

6. Voir notamment A. Batteur, A. Cerf-Hollender, A. Gosselin-Gorand, J.-M. Larralde, «Les interdits... », spéc. p. 226.

7. Le droit pénal appréhende depuis bien longtemps les atteintes à l'état de l'enfant. L'ancien droit connaissait déjà la « supposition » et la «suppression d'enfant", par exemple faire passer pour le sien un nouveau-né et faire disparaître un enfant sans pour autant lui enlever la vie. Voir R. Merle, A. Vitu, Traité de droit criminel, t. II, Droit pénal spécial, Paris, Cujas, 1982, nº 2146. L'article 345 du Code pénal de 1810 incriminait l'enlèvement, le recel, la suppression, la substitution et la supposition d'enfant.

8. Voir notamment le délaissement de mineur (art. 227-1 et 227-2 du Code pénal), ou d'une personne qui n'est pas en mesure de se protéger (art. 223-3 et 223-4 du Code pénal - Cass. crim., 23 février 2000, n 99-82817, Bulletin criminel, $\mathrm{n}^{\circ}$ 84, p. 245 : Revue de sciences criminelles, 2000, p. 610, obs. Y. Mayaud), la privation d'aliments ou de soins (art. 227-15 du Code pénal - Cass. crim., 12 octobre 2005, nº 05-81191, Bulletin criminel, n² 259, p. 907: Recueil Dalloz, 2006, p. 2446, note J.-Y. Maréchal; Gazette du Palais, 30 octobre-3 novembre 2005, avis D. Comaret; La semaine juridique, édition générale, $\mathrm{n}^{\circ}$ 7, 15 février 2006, II, 10022 et II, 10023, note J. Leblois-Happe; Droit pénal, nº 1, 2006, comm. 6, note M. Véron). 
d'un enfant», punis de 3 ans d'emprisonnement et de 45000 euros d'amende 9 .

Dans un contexte de mondialisation, le débat sur les prohibitions françaises a connu une vive résurgence puisqu'il est délicat de concilier prohibition et mondialisation. Les mouvements transfrontières ont conduit des couples stériles à mettre les autorités françaises devant ce qu'une auteure a appelé un «fait internationalement accompli $»^{10}$. Ces couples reviennent en France avec un enfant qui est issu d'une convention nulle au regard de notre droit interne. La question centrale est donc toujours la même puisqu'il s'agit de savoir si une situation créée à l'étranger sous l'empire d'une législation complaisante doit être reconnue ou non. S'est alors posée la question de la réception de ces gestations pour autrui réalisées à l'étranger par le droit français, à travers la question de la transcription en France des actes d'état civil des enfants nés à l'étranger.

Si dans un premier temps la Cour de cassation y était très hostile, elle a dû changer d'attitude notamment sous l'impulsion de la jurisprudence de la Cour européenne des droits de l'homme. Tout d'abord, ce fut l'époque de l'interdit. Le 6 avril 2011, la première chambre civile de la Cour de cassation a rendu trois arrêts concernant le sort en France des enfants conçus et nés à l'étranger en conformité avec le droit américain ${ }^{11}$. Les affaires ont en commun, même si les situations de fait et de droit ne sont pas tout à fait identiques, que les enfants concernés sont nés d'une mère porteuse en Californie. Pour la haute juridiction, la transcription, en France, de l'acte de naissance dressé en conséquence du jugement américain ou la reconnaissance d'une filiation fondée sur la possession d'état sont impossibles. La Cour se fonde, comme dans l'arrêt de 1991 de l'assemblée plénière, sur le principe de l'indisponibilité de l'état des personnes et considère que le processus est contraire à l'ordre public international français. Les arrêts ont été diversement accueillis et on a pu souligner qu'un ordre public international correspondant aux principes communs de l'humanité ou encore aux principes d'une justice universelle douée d'une valeur internationale absolue n'existe pas sur la question de la maternité pour autrui : les législations sont très partagées et évolutives ${ }^{12}$. Cette orientation de la jurisprudence a été confirmée par deux arrêts du 13 septembre $2013^{13}$ et un arrêt du 19 mars $2014^{14}$. Il y a néanmoins eu un changement de fondement puisque c'est la théorie de la fraude qui a été invoquée pour refuser la transcription de l'acte de naissance étranger alors même qu'était désigné le père biologique de l'enfant et non plus la notion d'ordre public international. Fraus omnia corrumpit. La théorie de la fraude apparaît dès lors comme un verrou supplémentaire à la reconnaissance en France de la filiation d'enfants nés d'une gestation pour autrui à l'étranger ${ }^{15}$.

Puis l'interdit fut relativisé. Ainsi, le Conseil d'État, tenant compte peut-être de la jurisprudence européenne, et se fondant sur l'intérêt supérieur de l'enfant, était plus réservé. Dans son arrêt du 4 mai $2011^{16}$, la haute juridiction administrative a considéré que la circonstance que la conception de ces enfants par $M$. A. et $\mathrm{M}^{\mathrm{me}} \mathrm{C}$. aurait pour origine un contrat entaché de nullité au regard de l'ordre public français serait, à la supposer établie, sans incidence sur l'obligation, faite à l'administration par les stipulations de l'article 3-1 de la Convention relative aux droits de l'enfant, d'accorder une attention primordiale à l'intérêt supérieur des enfants dans toutes les décisions les concernant. Certes, il ne s'agissait pas pour le Conseil d'État de se prononcer sur la transcription des actes sur les registres de l'état civil. Il a néanmoins considéré que le juge des référés pouvait enjoindre l'administration de délivrer un document de voyage permettant aux enfants concernés d'entrer sur le territoire national. Le garde des Sceaux s'est ému de la situation de ces enfants au regard de la nationalité française. Ainsi, la circulaire Taubira du 25 janvier 2013, validée par le Conseil d'État le 12 décembre $2014^{17}$, permet de délivrer aux enfants

9. Est aussi incriminée la "substitution » d'enfant, qui consiste à intervertir deux nourrissons juste après l'accouchement de leur mère respective, avant déclaration de leur naissance, afin d'intervertir aussi leur état civil. Voir A. Vitu, «La protection pénale de l'état civil de l'enfant», in Droit de l'enfant et de la famille: hommage à Marie-Josèphe Gebler, J.-F. Eschylle, C. Marraud (dir.), Nancy, Presses universitaires de Nancy, 1998, p. 95 sq.

10. Voir G. Salamé, Le devenir de la famille en droit international privé, Aix-en-Provence, Presses universitaires d'Aix-Marseille, 2006.

11. Cass, $1^{\text {re }}$ civ., 6 avril 2011, pourvois $\mathrm{n}^{\circ}$ 10-19053, 09-17130 et 09-66486: Recueil Dalloz, 2011, p. 1522, note D. Berthiau et L. Brunet; Droit de la famille, $\mathrm{n}^{\circ}$ 5, 2011, étude 14, note C. Neirinck; Revue critique de droit international privé, 2011, p. 722, note P. Hammje; Revue trimestrielle de droit civil, 2011, p. 340, obs. J. Hauser.

12. Droit de la famille, $\mathrm{n}^{\circ} 5,2011$, étude 14 , note C. Neirinck.

13. Cass., $1^{\mathrm{re}}$ civ., 13 septembre 2013, $\mathrm{n}^{\circ}$ 12-30138 et n ${ }^{\circ}$ 12-18315: Recueil Dalloz, 2013, p. 2349, chron. H. Fulchiron et C. Bidaud-Garon; Revue critique de droit international privé, 2013, p. 909, note P. Hammje; Revue trimestrielle de droit civil, 2013, p. 816, obs. J. Hauser; Journal du droit international, $\mathrm{n}^{\circ} 1$, 2014, comm. 1, note J. Guillaumé.

14. Cass., $1^{\text {re }}$ civ., 19 mars 2014, $\mathrm{n}^{\circ}$ 13-500005: Recueil Dalloz, 2014, p. 905, chron. H. Fulchiron et C. Bidaud-Garon; La semaine juridique, édition générale, nº 21-22, 26 mai 2014, p. 613, note J. Heymann; Revue critique de droit international privé, 2014, p. 619, note S. Bollée.

15. Ces décisions sont même apparues comme un durcissement de la jurisprudence puisque les situations visées mettent en cause la paternité du père d'intention qui est aussi le père biologique et seul initiateur du recours à une gestation pour autrui. Les éléments réunis par le ministère public caractérisant l'existence d'un processus frauduleux comportant une gestation pour le compte d'autrui, il en résulte une impossibilité de transcrire sur les registres de l'état civil français les actes de naissance des enfants. Il revient dès lors au législateur de prendre position car, en l'état du droit positif, les juges judiciaires considèrent que, lorsque la naissance est l'aboutissement, en fraude à la loi française, d'un processus d'ensemble comportant une convention de gestation pour autrui, le refus de transcription est justifié.

16. CE, réf., 4 mai 2011, $\mathrm{n}^{\circ} 348778$ : Droit de la famille, $\mathrm{n}^{\circ} 6,2011$, comm. 99, obs. C. Neirinck.

17. $\mathrm{CE}, 2^{\mathrm{e}} / 7^{\mathrm{e}} \mathrm{SSR}, 12$ décembre 2014, $\mathrm{n}^{\circ} 365779$, publié au Recueil Lebon. 
un certificat de nationalité française et d'éviter qu'ils ne soient apatrides ${ }^{18}$.

Quant à la Cour européenne des droits de l'homme, elle considère qu'il n'existe aucun consensus en Europe, ni sur la légalité de la gestation pour autrui, ni sur la reconnaissance juridique du lien de filiation entre les parents d'intention et les enfants ainsi légalement conçus à l'étranger, car ces questions suscitent dans les quarante-sept États membres de délicates interrogations d'ordre éthique ${ }^{19}$. Les États doivent en conséquence se voir reconnaître une ample marge d'appréciation dans leurs choix liés à la gestation pour autrui. Toutefois, cette marge d'appréciation se voit réduite dès lors qu'il est question de la filiation, car cela met en jeu un aspect essentiel de l'identité des individus. Il incombe en conséquence à la Cour de Strasbourg de vérifier si un juste équilibre a été ménagé entre les intérêts de l'État et ceux des individus directement touchés, eu égard notamment au principe essentiel selon lequel, chaque fois que la situation d'un enfant est en cause, l'intérêt supérieur de celui-ci doit primer. Concernant les parents, la Cour juge que la position adoptée par la Cour de cassation a permis de ménager un juste équilibre entre les intérêts des requérants et ceux de l'État, sans pour autant que cela compromette leur droit au respect de leur vie familiale. Elle ne retient donc aucune violation de l'article 8 de la Convention de ce point de vue. En revanche, le droit des enfants au respect de leur vie privée a été violé. En effet, la position des juridictions internes a conduit à porter atteinte à leur identité au sein de la société française, car la nationalité constitue un élément essentiel de l'identité de toute personne. Par ailleurs, le fait pour les enfants requérantes de ne pas être identifiées en droit français comme étant les enfants des requérants parents d'intention a des conséquences sur leurs droits sur la succession de ceux-ci. S'il est donc parfaitement "concevable que la France puisse souhaiter décourager ses ressortissants de recourir à l'étranger à une méthode de procréation qu'elle prohibe sur son territoire ${ }^{20}$, il est indéniable

[...] que les effets de la non reconnaissance en droit français du lien de filiation entre les enfants ainsi conçus et les parents d'intention ne se limitent pas à la situation de ces derniers, qui seuls ont fait le choix des modalités de procréation que leur reprochent les autorités françaises: ils portent aussi sur celle des enfants eux-mêmes, dont le droit au respect de la vie privée, qui implique que chacun puisse établir la substance de son identité, y compris sa filiation, se trouve significativement affecté ${ }^{21}$.

La Cour de Strasbourg juge ainsi que, compte tenu des conséquences des graves restrictions sur l'identité et le droit au respect de la vie privée des enfants requérantes, et qu'en faisant ainsi obstacle tant à la reconnaissance qu'à l'établissement en droit interne de leur lien de filiation à l'égard de leur père biologique, l'État défendeur a outrepassé sa marge d'appréciation. Le fait que le père d'intention soit le père biologique des enfants justifie d'autant plus la décision de la Cour européenne des droits de l'homme. Elle impose de prendre en compte la filiation biologique paternelle. La France est donc condamnée pour non-respect de la vie privée de ces enfants.

Dans ce contexte, la Cour de cassation ne pouvait plus maintenir sa jurisprudence antérieure et a opéré un revirement de jurisprudence dans deux arrêts d'assemblée plénière rendus le 3 juillet $2015^{22}$. L'acte de naissance n'étant ni irrégulier, ni falsifié et les faits qui y étaient déclarés correspondant à la réalité, la Cour de cassation considère que la convention de gestation pour autrui conclue entre $\mathrm{M}$. X et $\mathrm{M}^{\mathrm{me}} \mathrm{Z}$ ne faisait pas obstacle à la transcription de l'acte de naissance. Dans les deux affaires, le père d'intention était vraisemblablement le père biologique de l'enfant né d'une gestation pour autrui. Dans ces circonstances et dans ces seules circonstances, la transcription est autorisée. A contrario, et dans les cas où il n'y a que des parents d'intention, qui n'ont pas de lien biologique avec l'enfant, la transcription ne saurait être autorisée ${ }^{23}$. Ce faisant la Cour de cassation a respecté la jurisprudence de la Cour européenne des droits de l'homme en reconnaissant la filiation biologique paternelle. En revanche, la question de la maternité d'intention n'est pas abordée par ces arrêts car seuls des pères avaient demandé la transcription des actes de naissance de leurs enfants. Depuis, la France a de nouveau été condamnée par la Cour de Strasbourg ${ }^{24}$. Étaient en cause des enfants nés d'un père qui les avait reconnus et qui avait conclu une convention avec une mère porteuse indienne. La Cour européenne des droits de l'homme reprend la motivation et vise ses précédents arrêts Mennesson et Labassée.

Pourtant, la position de la Cour de Strasbourg est loin d'être limpide comme le révèle l'affaire Paradiso et Campanelli c. Italie concernant un couple ayant conclu une gestation pour autrui avec une clinique russe et qui s'est

18. Rappelons que la nationalité est un effet de la filiation: les enfants n'ont pas de filiation reconnue en France, mais ils peuvent avoir la nationalité française. Le risque d'apatridie est neutralisé. Leur vie quotidienne s'en trouve simplifiée. Par contre, ils n'ont pas d'autres droits attachés à la filiation comme des droits successoraux vis-à-vis de leurs parents d'intention.

19. Cour EDH, 26 juin 2014, Mennesson c. France, $\mathrm{n}^{\circ}$ 65192/11 et 26 juin 2014, Labassée c. France, n 65941/11.

20. Cour EDH, 26 juin 2014, Mennesson c. France, $\$ 99$.

21. Ibid.

22. Pourvois $n^{\circ} 14-21323$ et 15-500o2.

23. En ce sens, voir le communiqué publié par la haute juridiction qui précise que «[1]es espèces soumises à la Cour de cassation ne soulevaient pas la question de la transcription de la filiation établie à l'étranger à l'égard de parents d'intention: la Cour ne s'est donc pas prononcée sur ce cas de figure».

24. Cour EDH, 21 juillet 2016, Foulon et Bouvet c. France, no $9063 / 14$ et 10410/14. 
ensuite heurté au refus de l'enregistrement de l'enfant par les autorités italiennes. Dans un premier arrêt du 27 janvier $2015^{25}$, la Cour européenne des droits de l'homme (deuxième section) a jugé, conformément à sa position adoptée dans les arrêts Mennesson et Labassée, que les juges internes «n'ont pas pris une décision déraisonnable» en faisant une application stricte du droit national qui a eu pour conséquence la non-reconnaissance de la filiation établie à l'étranger du fait de l'absence de liens génétiques avec l'enfant ${ }^{26}$. Il n'existe donc pas de «droit » à la reconnaissance en droit interne du lien de filiation d'un enfant né d'une gestation pour autrui réalisée à l'étranger. Toutefois, les requérants (qui avaient vécu six mois avec l'enfant en Italie à partir de son troisième mois) peuvent se prévaloir de «liens familiaux de facto » relevant de la vie familiale au sens de l'article 8 de la Convention ${ }^{27}$. La Cour en conclut donc que le placement de l'enfant dans une famille d'accueil n'a pas «préservé le juste équilibre devant régner entre les intérêts en jeu», mais sans pour autant conclure que ce constat de violation de l'article 8 de la Convention oblige l'État à remettre le mineur aux requérants ${ }^{28}$. Saisie par voie de renvoi (art. 43 de la Convention) après l'arrêt de chambre du 27 janvier 2015, la Grande Chambre de la Cour de Strasbourg s'est à nouveau prononcée sur cette question dans un autre arrêt plus récent ${ }^{29}$. Elle y réaffirme clairement que le recours aux conventions de gestation pour autrui soulève des questions éthiques sensibles sur lesquelles il n'existe aucun consensus parmi les États contractants et retient une solution inverse. Relevant tout à la fois «l'absence de tout lien biologique entre l'enfant et les parents d'intention, la courte durée de la relation avec l'enfant et la précarité des liens du point de vue juridique», la Cour juge "que les conditions permettant de conclure à l'existence d'une vie familiale de facto ne sont pas remplies ${ }^{30}$, alors même qu'elle ne conteste ni l'existence d'un projet parental, ni la qualité des liens affectifs entre l'enfant et les requérants ${ }^{31}$. Rappelant l'ample marge d'appréciation dont jouissent les États pour les «sujets éthiquement sensibles» (adoption, prise en charge par l'État d'un enfant, procréation médicalement assistée et gestation pour autrui ${ }^{32}$ ), elle valide par ailleurs l'intervention des autorités italiennes pour limiter la filiation aux seuls liens biologiques ou d'adoption régulière, afin de préserver les enfants ${ }^{33}$. En effet,
Accepter de laisser l'enfant avec les requérants, peut-être dans l'optique que ceux-ci deviennent ses parents adoptifs, serait revenu à légaliser la situation créée par eux en violation de règles importantes du droit italien ${ }^{34}$.

Cet arrêt révèle donc un changement de position à l'égard de l'extension des vies familiales de facto et réaffirme la possibilité pour les États d'interdire les contrats commerciaux de gestation pour autrui. Ces deux arrêts illustrent sans aucun doute les fortes fractures existant au sein de la juridiction de Strasbourg sur cette question complexe ${ }^{35}$.

Le 5 juillet 2017, par cinq arrêts rendus le même jour, la Cour de cassation a conforté sa position en considérant que la convention de gestation pour autrui ne faisait pas obstacle à la transcription d'actes de l'état civil étrangers conformes à la réalité. Elle a donc bien distingué le cas de la filiation paternelle qui repose sur une réalité biologique, de celui de la mère d'intention. Elle a considéré que le refus de transcription de la filiation maternelle lorsque l'enfant est né à l'étranger à l'issue d'une gestation pour autrui résulte de la loi et poursuit un but légitime en ce qu'il tend à protéger l'enfant et la mère porteuse et vise à décourager cette pratique prohibée par les articles 16-7 et 16-9 du Code civil. La mère est celle qui accouche. Quand bien même les actes étrangers retiendraient pour mère cette mère d'intention, ces actes de naissance ne sont pas conformes à la réalité et ne sauraient être transcrits. La Cour a cependant ouvert la voie de l'adoption puisqu'elle a affirmé que la gestation pour autrui ne fait pas obstacle à l'adoption par le conjoint si les conditions légales sont remplies et si elle est conforme à l'intérêt de l'enfant ${ }^{36}$.

La question est donc sensible. L'interdit est indéniablement relativisé. Le corps se retrouve à nouveau pris dans une réflexion sur la manière dont le droit le saisit. Face à cette évolution, certains annoncent une fin de la prohibition de la gestation pour autrui sur le territoire national, voire une réflexion sur le régime juridique de ce nouveau contrat spécial... D'autres cependant pourraient y voir une sorte de renoncement devant un fait accompli, un alignement systématique sur le droit le plus permissif, une remise en cause de l'intérêt de l'État à voir respecter ses propres politiques publiques ou valeurs sociales.

25. Cour EDH, 27 janvier 2015, Paradiso et Campanelli c. Italie, $\mathrm{n}^{\circ}$ 25358/12: La semaine juridique, édition générale, $\mathrm{n}^{\circ}$ 7, 16 février $2015, \mathrm{p}$. 194, zoom F. Sudre.

26. Cour EDH, 27 janvier 2015, Paradiso et Campanelli c. Italie, $\$ 77$.

27. Ibid., $\$ 69$.

28. Ibid., $\$ 86-88$

29. Cour EDH, GC, 24 janvier 2017, Paradiso et Campanelli c. Italie, $\mathrm{n}^{\circ}$ 25358/12.

30. Ibid., $\$ 157$.

31. Ibid., $\$ 163$.

32. Ibid., $\$ 194$

33. Ibid., $\$ 177$.

34. Ibid., $\$ 215$

35. Voir J.-M. Larralde, «L'éloignement d'un enfant né illégalement d'un contrat de gestation pour autrui ne viole pas l'article 8 de la Convention européenne des droits de l'homme", L'essentiel droit de la famille et des personnes, $\mathrm{n}^{\circ}$ 3, 2017, p. 3 sq.

36. Cass., $1^{\text {re }}$ civ., 5 juillet $2017, \mathrm{n}^{\circ} 16-1690116-50025, \mathrm{n}^{\circ} 16-16495, \mathrm{n}^{\circ} 16-20052, \mathrm{n}^{\circ}$ 16-16455, $\mathrm{n}^{\circ} 15-28597$. 
Le rappel historique opéré montre que ce sont des questions épineuses, qui soulèvent d'âpres débats dont la portée s'étend bien au-delà des cercles universitaires. Alimentant les difficultés des conceptions contemporaines de la filiation $^{37}$, cette thématique soulève aussi des interrogations sur la protection de l'enfant mais aussi de la mère porteuse, sans parler du rôle de la volonté individuelle puisque c'est avant toute chose de techniques contractuelles dont il s'agit.

«Le corps entre liberté et propriété» : la préposition « entre» semble donc le mot essentiel du titre du colloque s'agissant de la gestation pour autrui. On pourrait même dire que le sujet est adapté à la culture normande dans la mesure où l'on se demande s'il faut maintenir la prohibition ou l'écarter ou tenter de concilier un antagonisme? Il serait présomptueux de dire que nous allons montrer comment sortir du guet. Toujours est-il que nous voudrions remettre dans cette thématique les enjeux des mots liberté (I) et propriété (II).

\section{La liberté}

L'article 4 de la Déclaration des droits de l'homme et du citoyen indique que

La liberté consiste à pouvoir faire tout ce qui ne nuit pas à autrui: ainsi, l'exercice des droits naturels de chaque homme n'a de bornes que celles qui assurent aux autres Membres de la Société la jouissance de ces mêmes droits. Ces bornes ne peuvent être déterminées que par la Loi.

Le phénomène étudié souligne donc la liberté de chaque individu de procréer et permet même d'élargir les formes de procréations. Se développe indéniablement une liberté de recourir aux techniques de la gestation pour autrui et des maternités de substitution. Les solutions du droit positif en relativisant l'interdit encouragent la liberté de recourir à ces techniques. Les derniers arrêts de la Cour de cassation abrogent de facto l'article 16-7 du Code civil «en tenant pour indifférente sa transgression $»^{38}$. Il serait même possible d'arguer qu'il devient absurde de maintenir une loi qu'un simple voyage permet d'éluder. Au-delà, on pourrait être tenté de considérer qu'il serait bon d'ouvrir la gestation pour autrui sous toutes ses formes sur le sol français en justifiant qu'elle serait très certainement mieux contrôlée en France que dans les États qui aujourd'hui sont ses terrains de prédilection, telle l'Inde ou encore les pays de l'Est de l'Europe. On pourrait cependant y voir une version inédite du gouvernement des juges. Quoi qu'il en soit, cette liberté remet en cause l'autorité de la loi et de la représentation nationale. Pourtant, le législateur peut se targuer du maintien de la prohibition dès lors que la société civile n'est pas prête à accepter ces techniques sur son territoire: l'engagement politique est maintenu.

Par ailleurs, les solutions du droit positif exacerbent une liberté pour le père biologique. Le père biologique apparaît comme le "grand gagnant » de cette jurisprudence. La Cour de cassation dans sa jurisprudence de 2015 se fonde sur la vérité factuelle des faits relatés dans l'acte de naissance étranger et insiste sur le fait que le revendiquant est bien le père biologique de l'enfant. L'acte de naissance n'étant ni irrégulier ni falsifié et les faits qui y étaient déclarés correspondant à la réalité, la Cour de cassation considère que la convention de gestation pour autrui conclue entre $M$. X et $M^{\text {me }} Z$ ne faisait pas obstacle à la transcription de l'acte de naissance. Dans les deux affaires, le père d'intention était considéré comme le père biologique de l'enfant né d'une gestation pour autrui. Dans ces circonstances et dans ces seules circonstances, la transcription est autorisée. Aucun test biologique n'a cependant été réalisé dans les espèces concernées... On pourrait être enclin à dire que ce n'est plus mater semper certa est mais pater semper certa est dès lors qu' un homme passe une convention de procréation avec une femme hors du territoire. La liberté du père biologique induit une liberté du processus de procréation puisque celui-ci n'est plus considéré. Pourtant, l'acte étranger peut être considéré comme porteur d'un état de droit et le for requis en ordonnant la transcription accepte de reconnaître cet état et de lui faire produire des effets. Néanmoins, ce n'est pas parce qu'une situation a été créée à l'étranger et qu'elle existe qu'elle devrait ipso facto être reconnue dans l'ordre juridique du for. C'est pour cela que la Cour de cassation avait un temps invoqué les critères d'ordre public et de fraude, lesquels sont exigés pour la réception des décisions étrangères sur le territoire national. La fraude permettait d'envisager le processus de création de l'enfant dans son ensemble ${ }^{39}$. On fait désormais fi du processus de création. Mais tous les mécanismes ne sont pas pour autant traités de la même manière. Ce sont les hypothèses où l'enfant est l'enfant biologique du père d'intention et que la mère porteuse est la mère biologique qui sont ainsi renforcées : majoritairement c'est donc le cas de la parentalité homosexuelle qui est visée ou encore de l'infertilité de la mère d'intention. Dans une telle logique, la mère d'intention est sacrifiée sur l'autel de la liberté: soit elle n'a pas donné ses gamètes et il n'y aura jamais la concernant de vérité biologique; soit elle a donné ses gamètes mais, en droit français, la mère est celle qui accouche et c'est donc la mère porteuse qui est la mère juridique. La maternité d'intention est exclue. Cela a été conforté par les arrêts du 5 juillet 2017 qui distinguent nettement le cas de la filiation paternelle de celui de la filiation maternelle. Le

37. Voir, à ce sujet, I. Théry, A.-M. Leroyer, Filiation, origines, parentalité: le droit face aux nouvelles valeurs de responsabilité générationnelle, rapport du groupe de travail Filiation, origines, parentalité au ministère des Affaires sociales et de la Santé, ministère délégué chargé de la Famille, 2014.

38. D. Sindres, «Brèves remarques sur les arrêts d'assemblée plénière du 3 juillet 2015 ouvrant la voie à la reconnaissance en France de la GPA", Recueil Dalloz, 2015, point de vue 31, p. 1773

39. Voir notamment D. Sindres, «Le tourisme procréatif et le droit international privé», Journal du droit international, $\mathrm{n}^{\circ} 2,2015, \mathrm{p} .429$ sq. 
droit positif permet donc d'évoquer une forme de liberté à deux vitesses. Seule une remise en cause des fondements de la filiation en droit français pourrait permettre un semblant d'égalité... ou encore une réelle compréhension de l'article $47 \mathrm{du}$ Code civil. Ce texte a pour objet de préciser les conditions auxquelles est présumée la force probante des actes de l'état civil étranger et d'expliciter les éléments permettant de renverser cette présomption. Sont ainsi mentionnés les actes irréguliers (ils ne respectent pas les formes locales), les actes falsifiés et les actes inexacts. L'article 47 exige donc une sorte de principe de véracité qui n'est pas à confondre avec les exigences d'ordre public international ou de fraude en droit international privé. Il est tout à fait possible d'avoir des actes qui respectent le principe de véracité en relatant des faits exacts mais que la situation relatée ait été créée au moyen d'une fraude au sens du droit international privé ou encore en heurtant la conception française de l'ordre public international. Or, l'ordre public international exige de bloquer la convention portant sur la création ou la gestation pour le compte d'autrui. C'est bien cette convention qui est à l'origine de la filiation biologique du père. Mais, si pour lui elle n'est pas prise en considération, pour la mère d'intention elle réapparaît. Pourtant, l'article 16-7 du Code civil n'interdit pas seulement les maternités de substitution. Il fait échec au recours par convention au service d'une mère porteuse qui portera l'enfant et l'abandonnera à sa naissance. L'article 16-7 du Code civil permet d'englober tous les cas, que cela soit un couple hétérosexuel, deux hommes, deux femmes, ou une personne célibataire de sexe masculin ou féminin.

Le phénomène permet ensuite d'évoquer la (prétendue ?) liberté de la mère porteuse: les mères porteuses sont essentiellement des femmes plus pauvres au service d'hommes et/ou de femmes plus riches. C'est un lieu commun de le dire mais qui ne peut être nié et doit être redit. La rémunération est omniprésente dans ces mécanismes invitant le marché ${ }^{40}$. Les conventions sont conclues à titre onéreux et ces femmes perçoivent souvent une part infime de ce qui est versé au profit des entremetteurs. Cela a pu mener à certaines dérives comme en Inde avec de véritables «élevages» de mères porteuses organisés et récemment interdits sous la pression de l'opinion publique mondiale. Or, si on fait fi du processus de création, on ferme les yeux sur toutes ces pratiques pas toujours bien identifiées telles que les stimulations ovariennes parfois réalisées susceptibles de porter atteinte à la santé de ces femmes.
Ces techniques de procréation conduisent aussi à considérer la liberté des sociétés de démarchage étrangères qui viennent sur le territoire proposer leurs services aux parents d'intention. Malgré la nullité sur le plan civil et l'interdit sur le plan pénal, le contexte de la mondialisation attire les sociétés étrangères, lesquelles par des démarches publicitaires exposent des données des pays permissifs. Il existe pourtant le délit d'entremise ${ }^{41}$. Des auteurs ont pu néanmoins montrer que notre arsenal pénal est bien peu efficace $^{42}$ face à ces démarchages. Si la loi pénale est applicable aux infractions commises sur le territoire (art. 113-2 du Code pénal), les sociétés entremetteuses se contentent de présenter dans le for les possibilités de gestation pour autrui et les options (choix du sexe de l'enfant, prix dégressif...) mais c'est à l'étranger que l'entremise, la mise en relation des parents d'intention et de la mère porteuse, a lieu. Certes, le fait constitutif pourrait être interprété largement et il pourrait être considéré que cela englobe les actes préparatoires et que ces actes préparatoires ont eu lieu en France. Par ailleurs, les principes de personnalité active (art. 113-6 du Code pénal) et de personnalité passive ne sont pas d'un grand secours: les infractions ne sont pas commises par des Français et, dès lors que l'on considère la victime, les choses se compliquent: soit il s'agit de considérer l'enfant mais au moment de l'infraction il n'existe pas, soit il s'agit de considérer les parents d'intention mais ils sont eux aussi à l'origine du processus, soit il s'agit de considérer la mère porteuse mais celle-ci, même si on peut la considérer victime de la marchandisation de son corps, n'est pas française. Le délit d'entremise apparaît une épée de Damoclès bien fragile au-dessus de la tête de ces sociétés.

"Le corps entre propriété et liberté»: la liberté des acteurs des techniques de gestation pour autrui est bien relative, particulièrement du point de vue des mères. Qu'en est-il maintenant de la propriété?

\section{La propriété}

Si on conçoit la propriété comme la relation que la personne est susceptible d'avoir avec tout intérêt susceptible de constituer un objet de droit rendant possible l'appropriation de l'homme et si on retient une conception large de cet objet en y incluant le corps humain, les processus de gestation pour autrui insufflent alors assurément un rôle nouveau à la propriété. Sont en l'occurrence objets de propriété l'enfant bien sûr mais aussi l'utérus de la mère porteuse.

40. Voir J. Icard, «Une analyse économique du droit de la famille. À propos de la gestation pour autrui», Revue de la recherche juridique. Droit prospectif, $\mathrm{n}^{\circ}$ 1, 2011, p. 131-152, spéc. p. 142 sq.; D. Le Breton, «La question anthropologique de la gestation pour autrui ", in Les incidences de la biomédecine sur la parenté, B. Feuillet-Liger, M.-C. Crespo-Brauner (dir.), Bruxelles, Bruylant, 2014, p. 337 sq.

41. Art. 227-12 du Code pénal: «Le fait de provoquer soit dans un but lucratif, soit par don, promesse, menace ou abus d'autorité, les parents ou l'un d'entre eux à abandonner un enfant né ou à naître est puni de six mois d'emprisonnement et de 7500 euros d'amende./ Le fait, dans un but lucratif, de s'entremettre entre une personne désireuse d'adopter un enfant et un parent désireux d'abandonner son enfant né ou à naitre est puni d'un an d'emprisonnement et de 15000 euros d'amende./ Est puni des peines prévues au deuxième alinéa le fait de s'entremettre entre une personne ou un couple désireux d'accueillir un enfant et une femme acceptant de porter en elle cet enfant en vue de le leur remettre. Lorsque ces faits ont été commis à titre habituel ou dans un but lucratif, les peines sont portées au double».

42. Voir B. Chapleau, «Le délit d'entremise en vue de la maternité pour autrui», Recueil Dalloz, 2015, p. 1775 sq. 
Bien entendu, dans cette hypothèse on doit faire table rase de l'article $16 \mathrm{du}$ Code civil ${ }^{43}$ pour l'enfant et de l'article suivant $16-1^{44}$ pour la mère porteuse. Levons ces obstacles, écartons ces textes de droit positif et admettons la marchandisation du corps et le «marché des ventres» et de l'enfant.

Autoriser les techniques de gestation pour autrui revient à reconnaître en la matière une certaine liberté contractuelle. Celles-ci ont d'ailleurs permis de montrer la combinaison de différents contrats, l'utilisation originale des contrats spéciaux. C'est une combinaison de contrats emportant d'une part la jouissance d'une chose et d'autre part le transfert de propriété d'une autre chose puisque le processus suppose non seulement le louage d'un utérus mais aussi le transfert de l'enfant né. Le louage d'utérus pourrait alors être envisagé comme un louage de chose ou plus vraisemblablement comme un louage d'ouvrage ou une prestation de service rémunérée...

À ce sujet, le vocabulaire du droit des affaires pénètre déjà le droit de la famille; la Cour de Luxembourg, ellemême, n'hésite pas à utiliser l'expression «mère commanditaire» pour désigner la mère d'intention. Elle considère que

[...] les États membres ne sont pas tenus d'accorder un congé de maternité [...] à une travailleuse, en sa qualité de mère commanditaire ayant eu un enfant grâce à une convention de mère porteuse, $\mathrm{y}$ compris lorsqu'elle est susceptible d'allaiter cet enfant après la naissance ou qu'elle l'allaite effectivement ${ }^{45}$.

Le processus libère un marché et ses forces qui tendent à transformer la personne en sujet économique. On utilise des mécanismes qui étaient réservés jusqu'alors aux stricts objets de droit pour les appliquer aux sujets de droit. Mais transformer des sujets de droit en objets de droit n'est pas si simple. Quelle valeur donner à la location d'un utérus ou au transfert de propriété d'un enfant? Quelle contrepartie peut être exigée? Est-ce un marché guidé par la loi de l'offre et de la demande? À l'évidence, ce ne peut pas être un marché comme les autres. Que l'on se place du point de vue de la mère porteuse ou de celui de l'enfant, des obstacles se lèvent contre cette marchandisation.

En ce qui concerne la mère porteuse, si l'on admet l'appropriation de son corps, on passe d'un régime de protection du corps humain à un régime de disponibilité qui transforme l'individu en propriétaire de son corps. Le processus est fondé sur la location d'un utérus. Au-delà, une réflexion peut être menée sur ce qui découle de cette location: l'impact du contrat sur la vie de cette femme, la régulation de son comportement, de sa vie sexuelle, de ses choix de modes de vie (alimentation, sport...). La propriété peut devenir aliénante... La jurisprudence actuelle utilise les notions d'intérêt de l'enfant, de droit à la vie privée des parents d'intention; mais il apparaît aussi que l'on doit envisager l'intérêt de cette mère porteuse. Les données actuelles qui laissent entendre que les arrêts récents ne donnent plus grand sens à la prohibition de la gestation pour autrui s'orientent alors vers un encadrement de celle-ci ${ }^{46}$. Cet encadrement sous-entend qu'il y a des risques pour la gestatrice, notamment pour sa santé en cas d'inséminations multiples. Certains évoquent des gestations pour autrui tolérables et des gestations pour autrui intolérables. Quoi qu'il en soit, elles révèlent inéluctablement une vision réductrice du corps de la femme alors que le corps n'est pas seulement un organisme. Il est aussi marqué par l'univers de la reproduction et il est imprégné de multiples données qui échappent au droit dans l'approche actuelle puisque la maternité n'est pas considérée dans sa globalité, la mère étant en droit positif «seulement» celle qui accouche.

Quant à l'enfant, il devient l'objet du processus. On parle de réification de l'enfant. Mais de quel enfant s'agitil ? Un enfant qui correspond en tous points aux attentes des parents d'intention telles qu'elles figurent au contrat. Que se passe-t-il si l'enfant ne correspond pas à ces attentes, notamment s'il naît handicapé ou s'il ne répond pas à tous les critères du parent ou des parents d'intention? Que deviendra l'enfant si nul n'en veut, ni le(s) parent(s) d'intention, ni la mère porteuse? Faudra-t-il le placer? Quel coût la prise en charge de cet enfant engendrera-t-elle

43. «La loi assure la primauté de la personne, interdit toute atteinte à la dignité de celle-ci et garantit le respect de l'être humain dès le commencement de sa vie.»

44. «Chacun a droit au respect de son corps. / Le corps humain est inviolable. / Le corps humain, ses éléments et ses produits ne peuvent faire l'objet d'un droit patrimonial.»

45. CJUE, 18 mars 2014, C. D. c. S. T., C-167/12, $\$ 43$. Une femme anglaise ayant eu un enfant grâce à une convention de mère porteuse comme l'autorise le droit anglais (Human Fertilisation and Embryology Act, 2008) et souhaitant l'allaiter avait sollicité de son employeur un congé payé à la suite de la naissance de cet enfant. Cette demande formelle de «congé de maternité de substitution » ayant été refusée, l'employeur considérant que le droit national ne prenait en compte que les congés de maternité ou les congés d'adoption, la Cour de justice devait vérifier la compatibilité de ce refus avec le directive 92/85/CEE du 19 octobre 1992 concernant la mise en œuvre des mesures visant à promouvoir l'amélioration de la sécurité et de la santé des travailleuses enceintes, accouchées ou allaitantes au travail et avec la directive 2006/54/CE du 5 juillet 2006 relative à la mise en œuvre du principe de l'égalité des chances et de l'égalité de traitement entre hommes et femmes en matière d'emploi et de travail. Si la Cour tend à préciser la situation des protagonistes en qualifiant de mère commanditaire la mère ayant eu un enfant grâce à une convention de mère porteuse et de mère porteuse la femme qui a été enceinte et a donné naissance à un enfant pour le compte d'une mère commanditaire, elle considère également qu'une travailleuse en sa qualité de commanditaire n'entre pas dans le champ d'application de la directive (art. 8) et que les États membres ne sont donc pas tenus d'accorder un congé de maternité à de telles travailleuses y compris lorsqu'elles sont susceptibles d'allaiter cet enfant après la naissance ou qu'elles l'allaitent effectivement. Le fait pour un employeur de refuser d'accorder un congé de maternité à une mère commanditaire ayant eu un enfant grâce à une convention de mère porteuse ne constitue pas davantage une discrimination fondée sur le sexe puisque les hommes ne peuvent pas non plus y prétendre.

46. Voir notamment, à ce sujet, M. André, A. Milon, H. de Richemont, Rapport d'information fait au nom de la commission des affaires sociales et de la commision des lois constitutionnelles, de législation, du suffrage universel, du Règlement et d'administration générale par le groupe de travail sur la maternité pour autrui, $\mathrm{n}^{\circ}$ 421, Sénat, déposé le 25 juin 2008. 
pour l'État? Jusqu'à combien la société est-elle prête à payer si l'enfant est abandonné? Allons encore plus loin: si l'enfant est réifié, devient objet de droit, à combien faut-il l'évaluer? Songeons aussi aux éventuels conflits entre le(s) parent(s) d'intention et la mère porteuse: si celle-ci veut en définitive garder l'enfant, qui l'emportera? Le perdant pourra-t-il être indemnisé? Pour quel préjudice? À quel quantum?

Pour conclure, il est vrai que le droit de la famille contemporain favorise indirectement cette marchandisation car il s'appuie fortement sur le «modèle du contrat» puisque ce modèle a permis de désinstitutionnaliser la famille, de considérer l'individu dans la famille, d'encourager la liberté et l'égalité au sein de la famille et a favorisé l'organisation de la famille par ses membres et non plus par l'État ${ }^{47}$. Cette mutation fondamentale affectant le droit de la famille emporte néanmoins des conséquences essentielles qui rejaillissent à l'occasion du débat sur la gestation pour autrui: le droit, et partant le droit de la famille, doit-il être une technique neutre d'organisation des relations familiales, un outil désymbolisé subordonné aux possibilités et aux finalités scientifiques ${ }^{48}$ ? Il s'agit alors de réfléchir aux rapports entre le droit et la science et de se demander si la norme scientifique doit être le seul critère de légitimité du droit. À ce titre, il faut se demander si l'assistance médicale à la procréation dans laquelle s'inscrivent ces techniques de gestation pour autrui peut intervenir pour simple convenance contrairement à ce qu'impose notre droit positif 49 . Plus généralement, le droit doit-il se lire dans les faits, les épouser, les suivre, coïncider avec eux, s'aligner sur eux, les calquer, les serrer au plus près, s'incliner devant eux, se soumettre? Nous ne le pensons pas, comme l'ont si magistralement montré Christian Atias et Didier Linotte dans leur précieux article, «Le mythe de l'adaptation du droit au fait $»^{50}$. Le droit n'est pas neutre.

47. Voir F. Dekeuwer-Défossez, «Réflexions sur les mythes fondateurs du droit contemporain de la famille», Revue trimestrielle de droit civil, 1995, p. 249-270.

48. Voir J. Icard, «Une analyse économique du droit de la famille... », spéc. p. 138.

49. Art. L. 2141-2 du Code de la santé publique: «L'assistance médicale à la procréation a pour objet de remédier à l'infertilité d'un couple ou d'éviter la transmission à l'enfant ou à un membre du couple d'une maladie d'une particulière gravité. Le caractère pathologique de l'infertilité doit être médicalement diagnostiqué. / L'homme et la femme formant le couple doivent être vivants, en âge de procréer et consentir préalablement au transfert des embryons ou à l'insémination. Font obstacle à l'insémination ou au transfert des embryons le décès d'un des membres du couple, le dépôt d'une requête en divorce ou en séparation de corps ou la cessation de la communauté de vie, ainsi que la révocation par écrit du consentement par l'homme ou la femme auprès du médecin chargé de mettre en œuvre l'assistance médicale à la procréation ».

50. C. Atias, D. Linotte, «Le mythe de l'adaptation du droit au fait», Recueil Dalloz, 1977, chron., p. 251. 